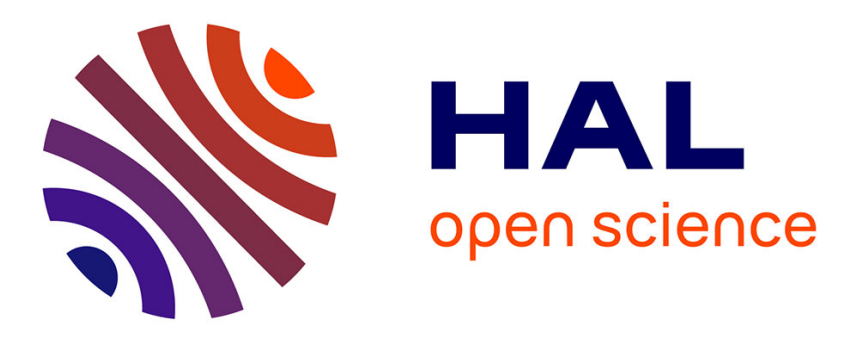

\title{
Near-Field Probe of Thermal Fluctuations of a Hemispherical Bubble Surface
}

Z. Zhang, Y. Wang, Y. Amarouchene, R. Boisgard, Hamid Kellay, A. Würger, A. Maali

\section{- To cite this version:}

Z. Zhang, Y. Wang, Y. Amarouchene, R. Boisgard, Hamid Kellay, et al.. Near-Field Probe of Thermal Fluctuations of a Hemispherical Bubble Surface. Physical Review Letters, 2021, 126 (17), 10.1103/PhysRevLett.126.174503 . hal-03255817

\section{HAL Id: hal-03255817 https://hal.science/hal-03255817}

Submitted on 9 Jun 2021

HAL is a multi-disciplinary open access archive for the deposit and dissemination of scientific research documents, whether they are published or not. The documents may come from teaching and research institutions in France or abroad, or from public or private research centers.
L'archive ouverte pluridisciplinaire HAL, est destinée au dépôt et à la diffusion de documents scientifiques de niveau recherche, publiés ou non, émanant des établissements d'enseignement et de recherche français ou étrangers, des laboratoires publics ou privés. 


\title{
Near-Field Probe of Thermal Fluctuations of a Hemispherical Bubble Surface
}

\author{
Z. Zhang $\odot,{ }^{1}$ Y. Wang $\odot,{ }^{2}$ Y. Amarouchene $\odot,{ }^{1}$ R. Boisgard, ${ }^{1}$ H. Kellay, ${ }^{1}$ A. Würger $\odot,{ }^{1}$ and A. Maali $\oplus^{1, *}$ \\ ${ }^{1}$ Université de Bordeaux \& CNRS, LOMA (UMR 5798), 33405 Talence, France \\ ${ }^{2}$ School of Mechanical Engineering and Automation, Beihang University, 37 Xueyuan Road, Haidian District, Beijing 100191, China
}

(Received 11 June 2020; accepted 30 March 2021; published 30 April 2021)

\begin{abstract}
We report measurements of resonant thermal capillary oscillations of a hemispherical liquid gas interface obtained using a half bubble deposited on a solid substrate. The thermal motion of the hemispherical interface is investigated using an atomic force microscope cantilever that probes the amplitude of vibrations of this interface versus frequency. The spectrum of such nanoscale thermal oscillations of the bubble surface presents several resonance peaks and reveals that the contact line of the hemispherical bubble is pinned on the substrate. The analysis of these peaks allows us to measure the surface viscosity of the bubble interface. Minute amounts of impurities are responsible for altering the rheology of the pure water surface.
\end{abstract}

DOI: 10.1103/PhysRevLett.126.174503

Introduction.-The static properties of liquid interfaces are to a large extent determined by the surface tension [1,2], whereas thermal fluctuations roughen the interfaces [3-13]. For finite-size interfaces such as millimeter-size droplets, the fluctuation spectrum consists of sharp resonance peaks at well-defined frequencies, which are related to liquid flow close to the surface [14]. In the case of droplets and bubbles deposited on a solid surface, the vibrational modes satisfy appropriate boundary conditions at the contact line.

Experimental studies of thermal capillary waves are mainly performed using techniques such as x-ray reflectivity [15], surface quasielastic light scattering [16,17], optical interferometry [18-20], and high speed video imaging [11,21]. Such techniques can also shed light on the viscoelastic properties of surfaces and interfaces when decorated by surfactants. These additives, even in minute quantities can alter not only the surface tension, but may result in a complex surface rheology and, in particular, induce surface elasticity and surface viscosity [22-32]. These latter quantities remain difficult to be measured [31]. For sessile droplets or bubbles, additional challenges arise from the contact line dynamics which has been investigated with methods like Marangoni flow, evaporation, and electrowetting [33-37]. Disturbing a sessile droplet with an atomic force microscope (AFM), McGuiggan et al. [38] observed the two lowest vibrational frequencies.

In this Letter we report on measurements of the thermal capillary oscillations on the surface of a bubble deposited on a solid substrate immersed in water. The hemispherical shape of the bubble with its contact line pinned on the substrate renders these oscillations resonant with welldefined peaks in their frequency spectrum; we have observed up to five peaks. The motion of the interface is studied using a cantilever that measures the vibrations' amplitude versus frequency. Our analysis of the frequencies, widths, and spectral weight of the thermally excited bubble vibrations shows that the contact line is indeed pinned and allows us to determine the surface viscosity at the bubble interface with a good precision.

Experimental setup.-Figure 1(a) shows the setup used in our experiments. A glass surface was covered using spin coating by a layer of polystyrene (PS) (thickness $100 \mathrm{~nm}$ and roughness $0.2 \mathrm{~nm}$ ). The air bubble was deposited on the PS surface in ultrapure water (Milli-Q, Direct-Q5, $18.2 \mathrm{M} \Omega \mathrm{cm}$ at $25^{\circ} \mathrm{C}$, Millipore Inc) using a microsyringe. The bubble was stable for several hours. During the experiment, we bring an atomic force microscope (AFM) cantilever in contact with the air bubble surface and measure its timedependent position, from which the spectral density of the

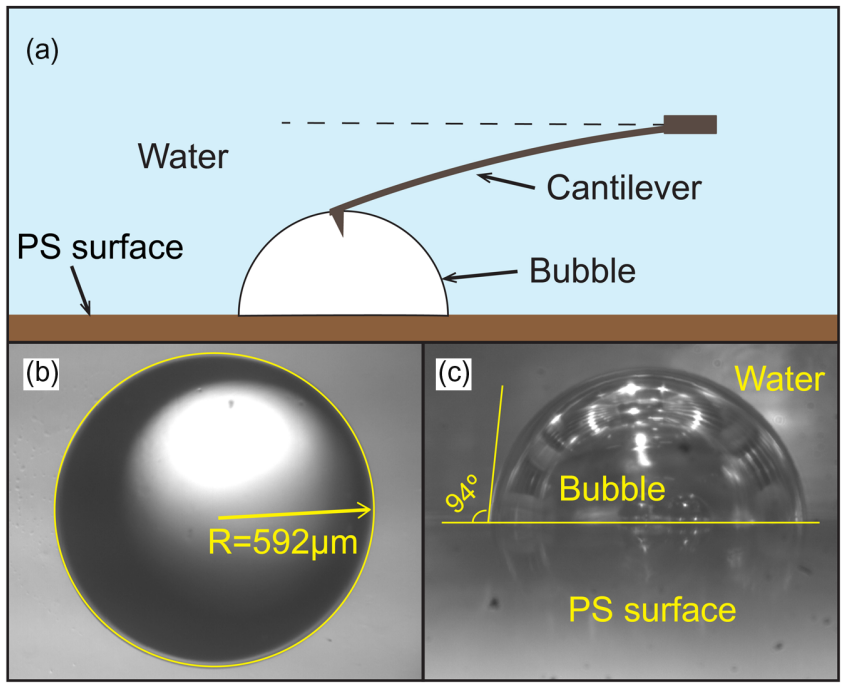

FIG. 1. (a) Experimental setup. The bubble was deposited on a polystyrene (PS) surface, and the cantilever tip was used to probe the vibration of the bubble. (b) Top view and (c) side view images, from which we obtain the bubble radius $R=592 \pm$ $5 \mu \mathrm{m}$ and the contact angle of $94 \pm 2^{\circ}$. 
capillary fluctuations of the water-air interface was determined. The radius $R$ and contact angle of the bubble were determined from the top view [Fig. 1(b)] and side view [Fig. 1(c)] optical images, respectively.

Experiments were performed using an AFM (Dimension 3100, Bruker) equipped with a liquid cell (DMFT-DD-HD) that allows operation in a liquid, and two different cantilevers (MLCT, Bruker, stiffness $k_{c}=0.024 \pm 0.002 \mathrm{~N} / \mathrm{m}$ and CSG01, NT-MDT, stiffness $k_{c}=0.12 \pm 0.02 \mathrm{~N} / \mathrm{m}$ ). The position of the cantilever was controlled by the AFM stepping motor stage allowing the cantilever to be brought into contact with the north pole of the bubble. Once this contact was established, the cantilever was driven solely by the vibrations of the bubble. The maximum amplitude of these vibrations was less than $1 \mathrm{~nm}$. The vertical deflection of the cantilever, due to these oscillations, was acquired by an A/D acquisition board (PCI-4462, National Instrument, USA). From the time series of the cantilever deflection signal, the power spectral density (PSD) of the AFM cantilever vibrations was calculated.

Resonance frequencies. - Typical PSD curves are shown in Fig. 2(a). The blue curve was measured for the cantilever in bulk water without bubble, showing the vibrational mode of the cantilever with characteristic frequency near $4 \mathrm{kHz}$. The main driving force for these cantilever fluctuations is the thermal noise [39-42]. The red curve depicts the PSD of the cantilever displacements when in contact with the bubble. Note that once the cantilever is in contact with the bubble surface, the cantilever resonance disappears and only the thermally activated modes of the bubble surface are observed. As a main finding, we observe well-defined resonance peaks for the cantilever in contact with the bubble, which we relate to thermal fluctuations of the bubble shape. At a frequency near $5.5 \mathrm{kHz}$, outside of the range of Fig. 2(a), the Minnaert resonance arising from volume oscillations of the bubble [43] can be observed (details are given in [44]). Coupling between the capillary oscillations and volume oscillations may occur when the resonance frequencies are very close. Care was taken to avoid such coupling by performing our analysis for resonance modes well below this peak.
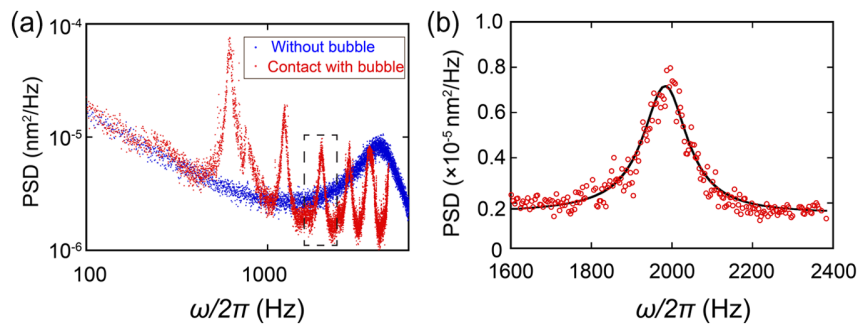

FIG. 2. Example of the measured PSD curves using a cantilever with stiffness $k_{c}=0.12 \mathrm{~N} / \mathrm{m}$. (a) The thermal spectra of the cantilever without the bubble (blue circles) and in contact with the bubble (red circles) deposited on PS surface. (b) The spectrum (circles) and the fitting curve using Eq. (3) (solid line) for the third peak in (a).
For a spherical inviscid liquid drop of surface tension $\sigma$, radius $R$, and fluid density $\rho$, Rayleigh predicted that the square of the resonance frequencies of the drop oscillations are multiples of [14]

$$
\omega_{0}^{2}=\frac{\sigma}{\rho R^{3}} .
$$

A similar relation occurs for the resonances of a gas bubble in a liquid [45]. For such drops or bubbles, each vibrational mode can be described as an oscillating string with an amplitude $\xi_{n}(t)$, satisfying the equation of motion

$$
m_{n}\left(\ddot{\xi}_{n}+2 \beta_{n} \dot{\xi}_{n}+\omega_{n}^{2} \xi_{n}\right)=\mathcal{F}_{n}(t),
$$

with the effective mass $m_{n}$, the damping coefficient $\beta_{n}$, the resonance frequency $\omega_{n}$ which is some multiple of $\omega_{0}$ and the mode number $n$. We use the shorthand notation $\dot{\xi}_{n}=d \xi_{n} / d t$. As we postulated above, the driving force $\mathcal{F}_{n}(t)$ is due to thermal noise, which is assumed uncorrelated in time and independent for each mode. Taking the Fourier transform of Eq. (2) and using the fluctuation dissipation theorem $\left|\mathcal{F}_{n}(\omega)\right|^{2}=2 \beta_{n} m_{n} k_{B} T$ [46], we obtain the one-sided power spectral density $S(\omega)=\sum_{n}\left|\xi_{n}(\omega)\right|^{2}$ in the form

$$
S(\omega)=\sum_{n \geq 1} \frac{4 \beta_{n}}{\left(\omega^{2}-\omega_{n}^{2}\right)^{2}+4 \beta_{n}^{2} \omega^{2}} \frac{k_{B} T}{\pi m_{n}} .
$$

Since the measured contact angle of our bubbles is very close to $90^{\circ}$, they may be considered hemispherical. The axisymmetric modes of a free bubble are described by Legendre polynomials of even degree, $P_{2 n}(\cos \theta)$ with the polar angle $\theta$ [14], which are labeled as $n=1,2,3, \ldots$.

The solutions of odd degree are not compatible with the presence of the solid boundary, since they would imply a finite velocity normal to the solid surface.

The vibrational spectrum depends on the boundary conditions for the oscillation amplitude at the solid surface. If the contact line moves freely along the surface, the capillary oscillations $\xi(t, \theta)=\sum_{n} \xi_{n}(t) P_{2 n}(\cos \theta)$ are simply the even modes of a spherical bubble in an inviscid liquid, where the natural frequencies $\hat{\omega}_{n}=\hat{\alpha}_{n} \omega_{0}$ are given by $\hat{\alpha}_{n}=\sqrt{(2 n-1)(2 n+1)(2 n+2)}[14,45,47]$. In Eq. (1) it is the density of the fluid surrounding the bubbles that is used $[14,44,47,48]$. For a pinned contact line, on the other hand, the nonslip boundary condition imposes the global constraint $\xi(t, \theta=\pi / 2)=0$. Then the vibration amplitude of a single mode is a superposition of even Legendre polynomials, $\xi_{n}(t)=\sum_{k} b_{n k} P_{2 k}(\cos \theta)$, with $b_{n k}<b_{n n}$. For contact angle $90^{\circ}$ Lyubimov et al. calculated the resonance frequencies $\omega_{n}=\alpha_{n} \omega_{0}$, where the coefficients $\alpha_{n}$ are solutions of the implicit equation $[38,44,49]$ 


$$
\sum_{k \geq 1} \frac{(2 k+1)(4 k+1)}{\hat{\alpha}_{k}^{2}-\alpha_{n}^{2}} P_{2 k}(0)^{2}=0
$$

The above description of bubble oscillations extended to the case of a half bubble resting on a solid surface with a contact angle close to $90^{\circ}$ can be compared quantitatively to the results of Fig. 2(a).

Figure 2(b) shows a fit to one of the peaks of the PSD using Eq. (3). Such fits, which account quantitatively for the shape of the peaks, allow us to determine the resonance frequency as well as the effective mass and the damping coefficient for the different mode numbers $n$. The values for the resonance frequencies normalized by $\omega_{0}$ obtained from the PSDs of different bubbles are plotted in Fig. 3(a). The results are best accounted for using nonslip boundary conditions; contact line pinning stiffens the vibrations and enhances the frequencies with respect to those obtained for slip boundary conditions, $\alpha_{n}>\hat{\alpha}_{n}$. Indeed, the comparison of Fig. 3(a) where the theoretical values for both nonslip and slip boundary conditions are displayed along with the experimental values, leads to the conclusion that the contact line of our bubble does not move on the surface but is pinned on the substrate. In the following we always refer to $\omega_{n}=\alpha_{n} \omega_{0}$.

Effective mass.-In Fig. 3(b) we plot the effective mass $m_{n}$ versus mode number $n$. These masses are extracted from fits of the PSD to Eq. (3). Here data from different bubble radii are displayed. Following Rayleigh [14], we express the kinetic energy through the velocity potential and integrate over the fluid volume $[44,47,50]$ to obtain the effective mass:

$$
m_{n}=\frac{2 \pi \rho R^{3}}{(2 n+1)(4 n+1)} .
$$

A comparison of Eq. (5) with the data for the measured effective mass $m_{n}$ is shown in Fig. 3(b). The effective mass has been normalized by $R^{3}$ giving a reasonable collapse of

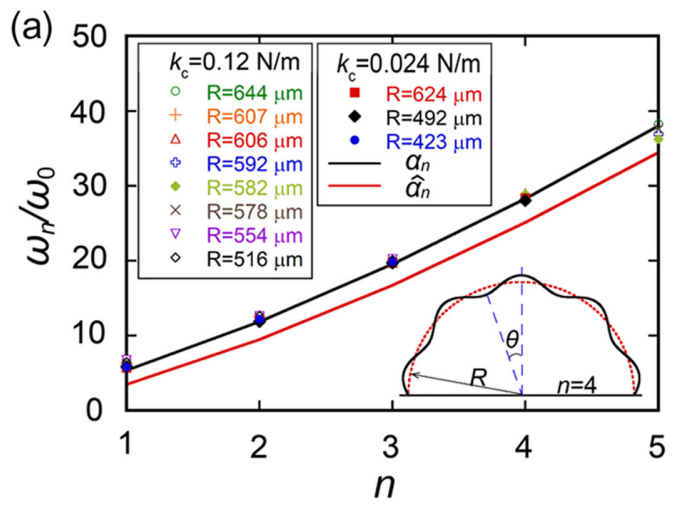

the data from different experiments using different bubble radii. Apart from the mode at $n=1$, the data from different realizations collapse on a single curve, and Eq. (5) accounts quantitatively for the decrease of this effective mass with the mode number $n$. The deviation at $n=1$ does not come as a surprise: Eq. (5) has been derived with the assumption of independent modes, which is plausible for higher modes but much less for the first one. Note that the relative difference between coupled and free modes, $\omega_{n}$ and $\hat{\omega}_{n}$, is significantly larger for $n=1$ (see Table S1 in Supplemental Material [44]).

Dissipation.-Now we turn to the damping coefficient $\beta_{n}$, which is directly related to the width of the peaks in Fig. 2(a). If the vibrational frequencies have been treated in the framework of potential flow of an inviscid fluid, damping arises from additional interactions. In previous studies, two mechanisms have been identified, which we refer to as viscous flow at the interface and at the solid boundaries, and surfactant contamination. At clean interfaces, viscous damping is the dominant source of dissipation [48], arising essentially from the flow in a surface layer of thickness $\delta=\sqrt{2 \eta / \rho \omega}$, where $\eta$ is the viscosity of the fluid surrounding the bubble. For frequencies in the kilohertz range, the penetration depth $\delta$ is of the order of ten microns, much smaller than the radius of our bubbles. In this case, Rayleigh's dissipation function is readily evaluated, resulting in the viscous damping coefficient $[44,47,51,52]$

$$
\beta_{n}^{\mathrm{vis}}=\frac{2 \eta}{\rho^{1 / 3} \sigma^{2 / 3}} \omega_{n}^{4 / 3}
$$

This expression does not account for dissipation on the solid substrate. In a study of Faraday waves [53] with nonslip boundary conditions at the container walls, Milner [24] showed that the increase of velocity within the penetration length $\delta$ results in a boundary contribution to the damping rate $\omega \delta / L$, which is inversely proportional to

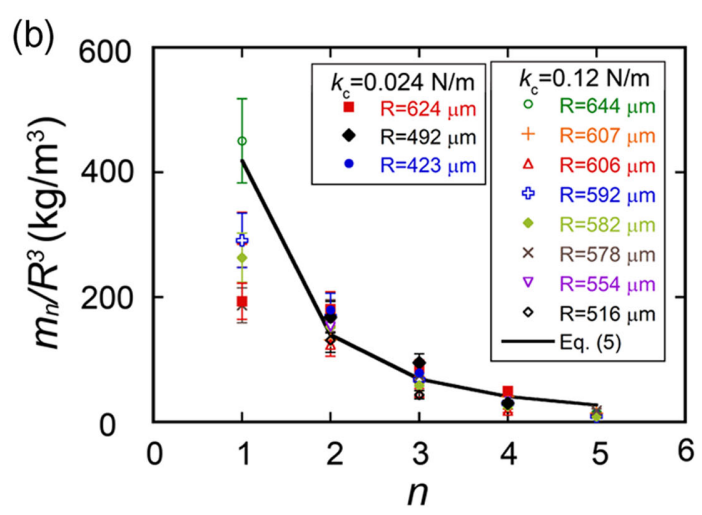

FIG. 3. (a) The results of the normalized resonance frequencies $\omega_{n} / \omega_{0}$ of the bubble versus the mode numbers. The black solid line connects the resonance frequency for pinned contact lines, and the red solid line for a freely moving contact line, as given in the main text. (b) The results of the effective mass normalized by the cubic power of the radius of the bubble versus the mode number for different bubbles. The dots with different colors and shapes represent the different measurements for different bubbles. The black line represents the theoretical results which was given by Eq. (5). 
the container size $L$. This leads us to estimate the boundary damping coefficient $\beta_{n}^{b}$ of the bubble oscillations as [44]

$$
\beta_{n}^{b}=\frac{3 \sqrt{2} \eta^{1 / 2}}{40 \rho^{1 / 2} R} \omega_{n}^{1 / 2}
$$

Besides the bulk-viscosity driven damping in (6) and (7), there are surface-induced contributions, arising either from an intrinsic surface viscosity [54] or from the contamination with surface-active agents $[22,25,31,32]$. The case of an infinite interface covered partially by contaminants, was studied by Miles, including surface viscosity and elasticity [22,32]. For the case of insoluble contaminants, Miles' theory provides the damping coefficient $\beta_{n}^{s}$ for capillary waves

$$
\beta_{n}^{s}=\frac{\sqrt{2} \eta^{1 / 2} \omega_{n}^{7 / 6}}{4 \rho^{1 / 6} \sigma^{1 / 3}} \frac{\hat{\chi}^{2}+\hat{\eta}(\hat{\eta}+2)}{(\hat{\chi}-1)^{2}+1+\hat{\eta}(\hat{\eta}+2)}
$$

with the dimensionless surface viscosity parameter $\hat{\eta}$ and elasticity parameter $\hat{\chi}$,

$$
\hat{\eta}=\frac{\sqrt{2} \rho^{1 / 6} \omega_{n}^{5 / 6}}{\eta^{1 / 2} \sigma^{2 / 3}} \eta_{s}, \quad \hat{\chi}=\frac{\sqrt{2} \rho^{1 / 6}}{\eta^{1 / 2} \sigma^{2 / 3} \omega_{n}^{1 / 6}} \chi .
$$

The surface viscosity $\eta_{s}$ includes both dilatation and shear viscosities, which cannot be distinguished in our experiment. The effective elasticity $\chi=\Gamma(d \sigma / d \Gamma)_{0}$ accounts for the tangential Marangoni stress induced by a change of the surfactant concentration $\Gamma$.

Figure 4 shows damping coefficients measured for different bubble radii. The data are plotted versus the resonance frequency. In the following we compare these

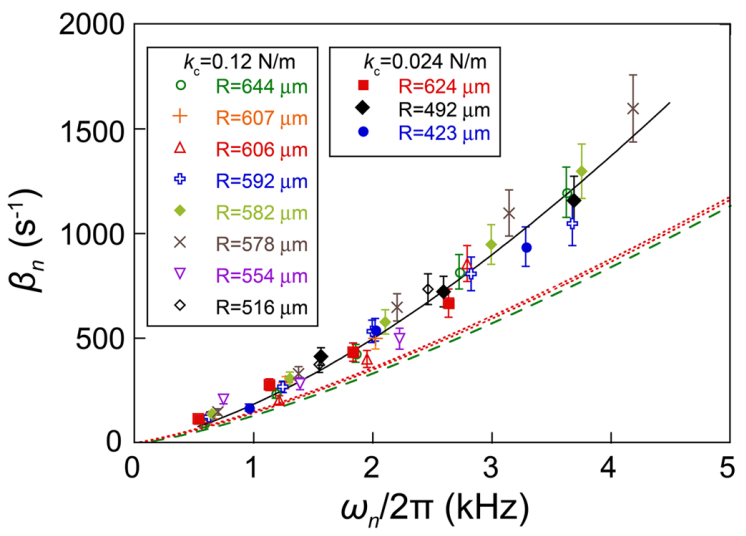

FIG. 4. Damping versus the frequency for different bubbles. The green dashed line corresponds to the viscous damping $\beta_{n}^{\mathrm{vis}}$ as in Eq. (6). The red dotted lines correspond to viscous damping plus boundary damping $\left(\beta_{n}^{\text {vis }}+\beta_{n}^{b}\right)$, with $\beta_{n}^{b}$ for $R=644$ and $423 \mu \mathrm{m}$. The black solid line is calculated from Eq. (10) and accounts for all terms in Eq. (10), including $\beta_{n}^{s}$ with the surface viscosity is $\eta_{s}=(1.5 \pm 0.2) \times 10^{-7} \mathrm{Pas} \mathrm{m}$. data in view of the theoretical result comprising both bulkviscosity and surface terms,

$$
\beta_{n}^{\mathrm{tot}}=\beta_{n}^{\mathrm{vis}}+\beta_{n}^{b}+\beta_{n}^{s} .
$$

Note that the only free parameters are the surface viscosity $\eta_{s}$ and elasticity $\chi$ appearing in Eq. (9).

We start with the viscous-flow contributions. The coefficient $\beta_{n}^{\text {vis }}$, plotted as a green dashed line in Fig. 4, captures rather well the overall trend yet is about a factor of 2 smaller than the experimental values. Adding the boundary term hardly changes the picture, as is obvious from the red dotted lines which give $\beta_{n}^{\text {vis }}+\beta_{n}^{b}$ for $R=644$ and $423 \mu \mathrm{m}$. Evaluating (6) and (7) with $\eta, \rho, \sigma$ of water, confirms the inequality $\beta_{n}^{b} \ll \beta_{n}^{\text {vis }}$. Since $\beta_{n}^{b}$ is the only term in (10) that depends explicitly on $R$, the above inequality also agrees with the observation that the data measured for different $R$, are independent of the bubble radius. Regarding the absolute value, we conclude that viscous damping is not sufficient to explain the measurements.

Surface viscosity.-The surface-related contribution to the damping coefficient $\beta_{n}^{s}$ depends both on surface viscosity and elasticity, in terms of the dimensionless parameters $\hat{\eta}$ and $\hat{\chi}$. Assuming that one or the other dominates, we performed two fits where either $\eta_{s}$ or $\chi$ is set to zero. The comparison in Fig. S3 [44] shows that $\chi=0$ and finite surface viscosity $\eta_{s}$, provide a much better description for the data, mainly because of the prefactors in (9) which result in quite a different frequency dependence. The black solid line in Fig. 4 depicts the total damping coefficient $\beta_{n}^{\text {tot }}$, by accounting for both bulk and surface viscosities, where the latter takes the value $\eta_{s}=(1.5 \pm 0.2) \times 10^{-7} \mathrm{~Pa} \mathrm{~s} \mathrm{~m}$.

In the above analysis, the surface viscosity is the only adjustable parameter. The different contributions to Eq. (10) are necessary for a satisfactory fit of the data from different experiments; the contribution of the surface viscosity is crucial for a better agreement with experimental values. The black solid line of Fig. 4 results mainly from the superposition of the viscous term $\beta_{n}^{\text {vis }} \propto \omega_{n}^{4 / 3}$ and the surface viscosity term, which behaves roughly as $\beta_{n}^{s} \propto \omega_{n}^{2}$ [44].

In order to verify that the flow created by the vibrating cantilever does not alter the dissipation and add an artifact in the damping coefficients, we have performed measurements with two cantilevers of different dimensions, one of stiffness $k_{c}=0.024 \mathrm{~N} / \mathrm{m}$, width $w=22 \mu \mathrm{m}$, length $l=208 \mu \mathrm{m}$, and a second one with $k_{c}=0.12 \mathrm{~N} / \mathrm{m}$, $w=34 \mu \mathrm{m}, l=350 \mu \mathrm{m}$. Because of the larger dimensions of the second cantilever, any effect of the cantilever beam on the damping coefficient should be significantly stronger than that of the first one. Yet Fig. 4 shows that there is no difference in the damping coefficients, which leads us to the conclusion that there is no unwanted backreaction of the vibrating cantilever on the bubble dynamics.

The fitted value of $\eta_{s}$ is about ten times larger than those reported by Earnshaw [54] for a pure water surface and Zell et al. [31] for soluble surfactant covered interfaces. 
The discrepancy could be due to the fact that in the present work, the surface viscosity measured accounts both for surface dilatational viscosity as well as surface shear viscosity. Further, we believe that our measurements are not devoid of surface contamination. In fact, in our previous study [55], despite the fact that a careful protocol was applied to minimize surface impurities, the air-water surface was found to be prone to contamination rather quickly with drastic effects on the properties of the water-air interface even for minute quantities of contaminants. We believe that there are similar effects here. Remarkably, our experimental technique is capable of probing the surface viscosity with a high precision. This is shown by Fig. 4 where the bulk effects are well below the measured damping rates. We hypothesize that coupling such a technique with precise techniques for measuring surface shear viscosities (such as that of Zell et al. [31]) provides a reliable technique to pin down the surface rheology of interfaces with various surface-active agents and disentangle dilatational from shear viscosities.

In conclusion, our experiments demonstrate that the AFM cantilever technique developed here is a powerful tool to probe the thermal capillary fluctuations of bubble surfaces. The spectrum of the fluctuations presents sharp resonance peaks for specific frequencies for which the motion of the interface is much more important than for other frequencies. Our measurements demonstrate that the contact line of a half bubble resting on a solid surface is pinned on the substrate and allows us to measure the additional damping due to the presence of minute amounts of contaminants. Moreover, our experimental method provides a useful new tool to probe the surface rheology.

Z.Z., R. B., A. W., and A. M. acknowledge the support by the French National Research Agency through grant No. ANR-19-CE30-0012. Z.Z. thanks the China Scholarship Council for financial support. Y.W. thanks the National Natural Science Foundation of China for financial support (Grants No. 51775028 and No. 52075029).

*Corresponding author. abdelhamid.maali@u-bordeaux.fr

[1] P.-G. De Gennes, F. Brochard-Wyart, and D. Quéré, Capillarity and Wetting Phenomena: Drops, Bubbles, Pearls, Waves (Springer-Verlag, New York, 2004).

[2] J. Rowlinson and B. Widom, Molecular Theory of Capillarity (Clarendon Press, Oxford, 1982).

[3] L. Turski and J. Langer, Dynamics of a diffuse liquid-vapor interface, Phys. Rev. A 22, 2189 (1980).

[4] M. Sferrazza, C. Xiao, R. A. L. Jones, D. G. Bucknall, J. Webster, and J. Penfold, Evidence for Capillary Waves at Immiscible Polymer/Polymer Interfaces, Phys. Rev. Lett. 78, 3693 (1997).
[5] J. Meunier, Liquid interfaces: Role of the fluctuations and analysis of ellipsometry and reflectivity measurements, J. Phys. 48, 1819 (1987).

[6] H. Kim, A. Rühm, L. Lurio, J. Basu, J. Lal, D. Lumma, S. Mochrie, and S. Sinha, Surface Dynamics of Polymer Films, Phys. Rev. Lett. 90, 068302 (2003).

[7] Y. Hennequin, D. G. A. L. Aarts, J. H. van der Wiel, G. Wegdam, J. Eggers, H. N. W. Lekkerkerker, and D. Bonn, Drop Formation by Thermal Fluctuations at an Ultralow Surface Tension, Phys. Rev. Lett. 97, 244502 (2006).

[8] D. Derks, D. G. Aarts, D. Bonn, H. N. Lekkerkerker, and A. Imhof, Suppression of Thermally Excited Capillary Waves by Shear Flow, Phys. Rev. Lett. 97, 038301 (2006).

[9] M. Fukuto, O. Gang, K. J. Alvine, and P. S. Pershan, Capillary wave fluctuations and intrinsic widths of coupled fluid-fluid interfaces: An x-ray scattering study of a wetting film on bulk liquid, Phys. Rev. E 74, 031607 (2006).

[10] A. M. Willis and J. B. Freund, Thermal capillary waves relaxing on atomically thin liquid films, Phys. Fluids 22, 022002 (2010).

[11] D. G. A. L. Aarts, M. Schmidt, and H. N. W. Lekkerkerker, Direct visual observation of thermal capillary waves, Science 304, 847 (2004).

[12] G. Michel, F. Pétrélis, and S. Fauve, Observation of Thermal Equilibrium in Capillary Wave Turbulence, Phys. Rev. Lett. 118, 144502 (2017).

[13] S. Perumanath, M. K. Borg, M. V. Chubynsky, J. E. Sprittles, and J. M. Reese, Droplet Coalescence is Initiated by Thermal Motion, Phys. Rev. Lett. 122, 104501 (2019).

[14] L. Rayleigh, On the capillary phenomena of jets, Proc. R. Soc. London 29, 71 (1879).

[15] B. M. Ocko, X. Z. Wu, E. B. Sirota, S. K. Sinha, and M. Deutsch, X-Ray Reflectivity Study of Thermal Capillary Waves on Liquid Surfaces, Phys. Rev. Lett. 72, 242 (1994).

[16] D. M. A. Buzza, General theory for capillary waves and surface light scattering, Langmuir 18, 8418 (2002).

[17] S. K. Peace, R. W. Richards, and N. Williams, Surface quasi-elastic light scattering from an amphiphilic graft copolymer at the air-water interface, Langmuir 14, 667 (1998).

[18] S. Maayani, L. L. Martin, S. Kaminski, and T. Carmon, Cavity optocapillaries, Optica 3, 552 (2016).

[19] L. Cantu, A. Raudino, and M. Corti, An interferometric technique to study capillary waves, Adv. Colloid Interface Sci. 247, 23 (2017).

[20] B. Pottier, C. Frétigny, and L. Talini, Boundary Condition in Liquid Thin Films Revealed through the Thermal Fluctuations of Their Free Surfaces, Phys. Rev. Lett. 114, 227801 (2015).

[21] G. Bolognesi, Y. Saito, A. I. Tyler, A. D. Ward, C. D. Bain, and $\mathrm{O}$. Ces, Mechanical characterization of ultralow interfacial tension oil-in-water droplets by thermal capillary wave analysis in a microfluidic device, Langmuir 32, 3580 (2016).

[22] J. W. Miles, Surface-wave damping in closed basins, Proc. R. Soc. A 297, 459 (1967).

[23] C. Stenvot and D. Langevin, Study of viscoelasticity of soluble monolayers using analysis of propagation of excited capillary waves, Langmuir 4, 1179 (1988). 
[24] S. T. Milner, Square patterns and secondary instabilities in driven capillary waves, J. Fluid Mech. 225, 81 (1991).

[25] H.-L. Lu and R. E. Apfel, Shape oscillations of drops in the presence of surfactants, J. Fluid Mech. 222, 351 (1991).

[26] T. Chou, S. Lucas, and H. A. Stone, Capillary wave scattering from a surfactant domain, Phys. Fluids 7, 1872 (1995).

[27] T. Asaki, D. Thiessen, and P. Marston, Effect of an Insoluble Surfactant on Capillary Oscillations of Bubbles in Water: Observation of a Maximum in the Damping, Phys. Rev. Lett. 75, 2686 (1995).

[28] J. T. Petkov, K. D. Danov, N. D. Denkov, R. Aust, and F. Durst, Precise method for measuring the shear surface viscosity of surfactant monolayers, Langmuir 12, 2650 (1996).

[29] C. Barentin, C. Ybert, J.-M. Di Meglio, and J.-F. Joanny, Surface shear viscosity of Gibbs and Langmuir monolayers, J. Fluid Mech. 397, 331 (1999).

[30] J. Giermanska-Kahn, F. Monroy, and D. Langevin, Negative effective surface viscosities in insoluble fatty acid monolayers: Effect of phase transitions on dilational viscoelasticity, Phys. Rev. E 60, 7163 (1999).

[31] Z. A. Zell, A. Nowbahar, V. Mansard, L. G. Leal, S. S. Deshmukh, J. M. Mecca, C. J. Tucker, and T. M. Squires, Surface shear inviscidity of soluble surfactants, Proc. Natl. Acad. Sci. U.S.A. 111, 3677 (2014).

[32] G. K. Rajan and D. M. Henderson, Linear waves at a surfactant-contaminated interface separating two fluids: Dispersion and dissipation of capillary-gravity waves, Phys. Fluids 30, 072104 (2018).

[33] D. Bonn, J. Eggers, J. Indekeu, J. Meunier, and E. Rolley, Wetting and spreading, Rev. Mod. Phys. 81, 739 (2009).

[34] R. V. Craster and O. K. Matar, Dynamics and stability of thin liquid films, Rev. Mod. Phys. 81, 1131 (2009).

[35] F. Mugele and J.-C. Baret, Electrowetting: From basics to applications, J. Phys. Condens. Matter 17, R705 (2005).

[36] P. Roach, N. J. Shirtcliffe, and M. I. Newton, Progess in superhydrophobic surface development, Soft Matter 4, 224 (2008).

[37] J. H. Snoeijer and B. Andreotti, Moving contact lines: Scales, regimes, and dynamical transitions, Annu. Rev. Fluid Mech. 45, 269 (2013).

[38] P. M. McGuiggan, D. A. Grave, J. S. Wallace, S. Cheng, A. Prosperetti, and M. O. Robbins, Dynamics of a disturbed sessile drop measured by atomic force microscopy (AFM), Langmuir 27, 11966 (2011).
[39] H.-J. Butt and M. Jaschke, Calculation of thermal noise in atomic force microscopy, Nanotechnology 6, 1 (1995).

[40] R. Levy and M. Maaloum, Measuring the spring constant of atomic force microscope cantilevers: Thermal fluctuations and other methods, Nanotechnology 13, 33 (2001).

[41] L.-O. Heim, T. S. Rodrigues, and E. Bonaccurso, Direct thermal noise calibration of colloidal probe cantilevers, Colloids Surf. A 443, 377 (2014).

[42] B. Hauer, C. Doolin, K. Beach, and J. Davis, A general procedure for thermomechanical calibration of nano/micromechanical resonators, Ann. Phys. (Amsterdam) 339, 181 (2013).

[43] M. Minnaert, XVI. On musical air-bubbles and the sounds of running water, London, Edinburgh, Dublin Philos. Mag. J., Sci. 16, 235 (1933).

[44] See Supplemental Material at http://link.aps.org/ supplemental/10.1103/PhysRevLett.126.174503 for technical details of data treatment and theory.

[45] A. Prosperetti, Free oscillations of drops and bubbles: the initial-value problem, J. Fluid Mech. 100, 333 (1980).

[46] R. Kubo, The fluctuation-dissipation theorem, Rep. Prog. Phys. 29, 255 (1966).

[47] A. Prosperetti, Linear oscillations of constrained drops, bubbles, and plane liquid surfaces, Phys. Fluids 24, 032109 (2012).

[48] H. Lamb, Hydrodynamics (Cambridge University Press, Cambridge, 1916).

[49] D. V. Lyubimov, T. P. Lyubimova, and S. V. Shklyaev, Behavior of a drop on an oscillating solid plate, Phys. Fluids 18, 012101 (2006).

[50] J. Vejrazka, L. Vobecka, and J. Tihon, Linear oscillations of a supported bubble or drop, Phys. Fluids 25, 062102 (2013).

[51] J. S. Sharp, Resonant properties of sessile droplets; contact angle dependence of the resonant frequency and width in glycerol/water mixtures, Soft Matter 8, 399 (2012).

[52] L. D. Landau and E. M. Lifshitz, Fluid Mechanics, Course of Theoretical Physics (Pergamon Press, Oxford, 1959).

[53] M. Faraday, On the forms and states assumed by fluids in contact with vibrating elastic surfaces, Phil. Trans. R. Soc. London 121, 299 (1831).

[54] J. C. Earnshaw, Surface viscosity of water, Nature (London) 292, 138 (1981).

[55] A. Maali, R. Boisgard, H. Chraibi, Z. Zhang, H. Kellay, and A. Würger, Viscoelastic Drag Forces and Crossover from No-Slip to Slip Boundary Conditions for Flow near AirWater Interfaces, Phys. Rev. Lett. 118, 084501 (2017). 